Artículo

\title{
Análisis de crecimiento en sorgo forrajero en dos períodos de siembra
}

\author{
Alejandra Pérez Hernández ${ }^{1}$ \\ Adrián Raymundo Quero Carrillo ${ }^{1 \S}$ \\ Santiago Garduño Velázquez ${ }^{2}$ \\ José Alberto Salvador Escalante Estrada ${ }^{1}$ \\ María Teresa Rodríguez González ${ }^{1}$ \\ Leonor Miranda Jiménez ${ }^{1}$
}

${ }^{1}$ Colegio de Postgraduados en Ciencias Agrícolas-Campus Montecillo. Carretera México-Texcoco km 36.5, Estado de México, México. CP. 56230. Tel. 595 9520279. (bawer29@hotmail.com; jasee@colpos.mx; mate@colpos.mx; miranda_leonor@yahoo.com). ${ }^{2}$ Universidad Autónoma Chapingo. Carretera MéxicoTexcoco km 38.5, Estado de México, México. CP. 56227. (santiago432@hotmail.com).

${ }^{\S}$ Autor para correspondencia: queroadrian@ @otmail.com.

\section{Resumen}

Análisis de crecimiento es una cuantificación periódica del rendimiento en materia seca que permite, determinar el momento óptimo de corte. El objetivo fue conocer el crecimiento, para cuantificar la acumulación de materia seca en un intervalo de tiempo, en cuatro variedades de sorgo forrajero: Caña dulce, Silo miel, Esmeralda y Fortuna, similarmente, determinar fenología, en dos períodos de siembra: punta de riego (abril-octubre) y lluvias (junio-diciembre) en Montecillo, Texcoco, Estado de México. Se determinó fenología, altura de planta (AP), número de hojas (NDH), número de tallos (NDT), número de nudos por planta (NDP), rendimiento de materia seca (RMS), se calculó índice de área foliar (IAF), duración de área foliar (DAF), tasa de crecimiento del cultivo (TCC) y tasa de asimilación neta (TAN). El diseño experimental bloques completos al azar con arreglo factorial y cuatro repeticiones, variables significativas, se compararon con prueba de Tukey $(p \leq 0.05)$. Caña dulce presentó mayor AP $(210 \mathrm{~cm})$ en ambos períodos, a 145 días después de la siembra(dds) promedio; NDT (33), NDH (8.1), NDN (8.3); TCC (24 g m $\mathrm{g} \mathrm{d}^{-1} \mathrm{a}$ 75(dds) y $26 \mathrm{~g} \mathrm{~m}^{-2} \mathrm{~d}^{-1}$ a $\left.84 \mathrm{dds}\right)$, TAN (19.1 $\mathrm{g} \mathrm{m}^{-2} \mathrm{~d}^{-1}$ y $13.8 \mathrm{~g} \mathrm{~m}^{-2} \mathrm{~d}^{-1}$ desde emergencia a $\left.20 \mathrm{dds}\right)$; lo anterior, resultó en mayores rendimientos $(p \leq 0.05)$ por metro cuadrado (15.7 y $\left.15.5 \mathrm{t} \mathrm{Ms} \mathrm{ha}^{-1}\right)$ en punta de riego y período de lluvias, respectivamente. El cultivar Silo miel presentó mayor IAF con 5 a 125 dds y 4.6 a 120 dds y DAF de 114 días; lo anterior, para punta de riego y lluvias, respectivamente. El mejor cultivar para la región fue Caña dulce.

Palabras clave: Sorghum bicolor (L.) Moench, fenología, lluvias, rendimiento.

Recibido: marzo de 2019

Aceptado: junio de 2019 


\section{Introducción}

El sorgo Sorghum bicolor (L.) Moench, es un cultivo cada vez más utilizado como forraje, en México en el ciclo agrícola 2017 se sembraron 192 mil ha de sorgo forrajero con rendimientos pomedio de $20 \mathrm{t} \mathrm{MS} \mathrm{ha}^{-1}$ (SIAP, 2019), esto se debe a su elevada tolerancia a sequía, especialmente en etapa vegetativa (Castro et al., 2000), se ha adaptado mejor que el maíz, debido que produce mayor cantidad de materia seca (MS) por unidad de agua consumida, una planta de sorgo consume de 80 a 100 mililitros menos que maíz en etapa reproductiva (Ajeigbe et al., 2018).

El sorgo evita la deshidratación debido a la cubierta serosa en hojas y por regulación osmótica, aumenta la absorción de agua a través de su sistema radical fibroso, denso y ramificado, que permite tener gran exploración del suelo y absorción de nitrógeno (Legarto, 2000) además, el sorgo frena la pérdida transpiracional de agua a través del hábito de crecimiento vertical de las hojas, tolera alta salinidad y manejo intensivo de cortes (Rouf et al., 2018); lo anterior, debido a sus ventajas con respecto a otros cultivos forrajeros como maíz, alfalfa, avena, etc; sin embargo, en suelo fértil y con disponibilidad de riego, el sorgo no compite en rendimiento de materia seca con maíz ni alfalfa, se adapta mejor a ambientes más desafiantes respecto a estos cultivos.

Existe poca información de sorgos forrajeros en el Altiplano Central de México (Bolaños et al., 2013), los estudios realizados son principalmente en rendimiento de materia seca (Borghi et al., 2013); sin embargo, para comprender mejor los factores fisiologicos que determinan el rendimiento y diferencias entre variedades, se requieren estudios detallados del crecimiento vegetal que permitan identificar y cuantificar la distribución de materia seca en etapas fenológicas, duración del ciclo de cultivo y determinar el momento óptimo de corte para producción de forraje (Egli, 2017; Pérez et al., 2017). La fenología se refiere al estudio de eventos biológicos involucrados en la vida de las plantas, como emergencia, amacollamiento, floración, fructificación, maduración y la interacción de estas etapas con el clima (Granados y Sarabia, 2013).

El ciclo de cultivo cambia entre variedades, esto quiere decir que diferentes variedades sembrados en el mismo sitio pueden presentar diferentes estados de desarrollo, después de transcurrido el mismo tiempo cronológico, por lo tanto, las prácticas de manejo deben adecuarse a la fenología de los cultivos (Solórzano, 2007).

En cuanto al análisis de crecimiento, es básico para comprender procesos físiológicos que determinan la producción de materia seca y racionalizar las prácticas de manejo (Azam, 2013), así como la fertilización para aumentar la eficiencia del cultivo, dando mejores índices de crecimiento, tales como tasa de crecimiento del cultivo (TCC), tasa media de asimilación neta (TAN), índice de área foliar (IAF) y duración del área foliar (DAF; Hunt, 1990; Escalante y Kohashi, 2015). Al respecto Gaytán et al. (2001), mencionan que variedades con altos índices de TCC, TAN e IAF presentan mayor translocación en formación de estructuras reproductivas, que repercute en peso seco total, estos índices pueden permitir determinar diferencias entre variedades de la misma especie y seleccionar los adecuados (Bednarz et al., 2000).

El objetivo fue conocer la fenología, mediante análisis de crecimiento y cuantificación del rendimiento de materia seca en cuatro variedades de sorgo forrajero, con dos fechas de siembra: punta de riego (abril-octubre) y período de lluvias (junio-diciembre) en Montecillo, Estado de México. 


\section{Materiales y métodos}

Las parcelas experimentales se localizaron en el área experimental del Colegio de Postgraduados Campus Montecillo, Texcoco, Estado de México (19²9' latitud norte, $98^{\circ} 51^{\prime}$ longitud oeste, 2 $250 \mathrm{msnm}$ ), clima $\mathrm{BS}_{1}$, el menos seco de los áridos con lluvias en verano, temperatura media anual $14.6{ }^{\circ} \mathrm{C}, 559 \mathrm{~mm}$ de precipitación (García, 2005). Asimismo, el suelo del lugar es de tipo alcalino, $\mathrm{pH}$ 9, CE $3.44 \mathrm{dS} \mathrm{m}^{-1}$ y MO 1.29\% y textura franco-arcillosa. El presente estudio se llevó a cabo en dos fechas de siembra; lo anterior, debido que existe amplia variabilidad en temperatura y distribución de la precipitación pluvial durante un año. El primer período (punta de riego), se sembró el 12 de abril, con un único riego $(40 \mathrm{~mm})$ que permitió la germinación y emergencia de las plantas, el segundo período (lluvias), se sembró el 17 de junio, ambos en el año 2013.

Los tratamientos consistieron en una variedad local (Caña dulce, empresa Berentsen) y tres variedades utilizados en el estado de Jalisco [Silo miel (Genex), Esmeralda (ABT) y Fortuna (INIFAP)], todos de uso forrajero. La extensión de la parcela donde se elaboró el experimento fueron $1000 \mathrm{~m}^{2}$. La siembra se realizó manualmente con deposito continuo de semillas (chorrillo), distancia entre surcos $70 \mathrm{~cm}$ y a densidad de $25 \mathrm{~kg} \mathrm{ha}^{-1}$ de SPV, posteriormente 25 días después de la siembra (dds) se realizó aclareo, hasta obtener una densidad de 17 plantas $\mathrm{m}^{-2}$, después a 40 dds, se fertilizó a razón de $80 \mathrm{~kg} \mathrm{~N} \mathrm{ha}^{-1}$, en el aporque. Durante el ciclo de crecimiento del cultivo, se registró temperatura media semanal máxima, mínima y suma semanal de precipitación.

Las unidades experimentales estuvieron constituidas de cinco surcos de $5 \mathrm{~m}$ de largo, de los cuales, como parcela útil se tomaron $3 \mathrm{~m}$ de tres surcos centrales. El diseño experimental fue bloques completos al azar y arreglo factorial con cuatro repeticiones, donde se tomó en cuenta cuatro variedades, cinco fechas de muestreo y dos períodos de siembra. Se realizó análisis de varianza y prueba de Tukey $(p \leq 0.05)$, en variables que resultaron significativas, con el paquete para análisis estadístico InfoStat (InfoStat, 2008).

\section{Fenología}

Las etapas fenológicas, se registraron en días después de la siembra (dds), debido a que la medición fue visual (Solórzano, 2007): vegetativas (V-1= emergencia, V-2= tercer hoja ligulada, V-3= quinta hoja ligulada, V-4= octava hoja ligulada, V-5= hoja bandera visible) y reproductivas (R-6= panícula extendida dentro de la vaina de hoja bandera, $\mathrm{R}-7=$ floración, $\mathrm{R}-8=$ grano masoso suave, R-9= grano masoso duro), los datos se registraron cuando $50 \%$ de la población presentó las características mencionadas.

\section{Variables de crecimiento}

Se registraron mediante evaluaciones periódicas y destructivas de un macollo por unidad experimental; en mediciones altura de planta (AP), número de hojas (NDH) y número de nudos (NDN), la medición se realizó en el tallo principal, AP se cortó a ras de suelo, el tallo y se midió con regla graduada, desde la parte basal hasta la última hoja o inflorescencia. NDH se contabilizaron al momento de remover hojas y vainas para exponer los nudos del tallo (NDN) y realizar conteo. NDT se realizó al momento de cortar el macollo. Todas las mediciones se realizaron a 25, 55, 85, 115 y 145 dds, también se calculó IAF, DAF, TCC y TAN. 
El rendimiento de materia seca (RMS; $\mathrm{t} \mathrm{MS} \mathrm{ha-1),} \mathrm{se} \mathrm{obtuvo} \mathrm{al} \mathrm{cortar} \mathrm{a} \mathrm{ras} \mathrm{del} \mathrm{suelo,} \mathrm{después} \mathrm{el}$ material vegetal se colocó sobre mesas de trabajo y se separó en sus componentes morfológicos y las láminas foliares fueron pasadas por un integrador de ares foliar LI-COR 3100, después cada componente se colocó en bolsas de papel debidamente rotuladas y permanecieron durante $72 \mathrm{~h}$ en estufa de circulación forzada de aire, a $70{ }^{\circ} \mathrm{C}$, posteriormente se retiró y peso en balanza analítica y se transformó t MS ha"-1.

Los cálculos se realizaron en intervalos de corte: 0-25, 26-55, 56-85 y 86-115 dds, incluyen IAF, DAF, TAN y TCC; para lo cual, se utilizaron las fórmulas de Escalante y Kohashi (2015): $\mathrm{IAF}=\left[(\mathrm{AF} / \mathrm{NP}) *\left(\mathrm{DP} / 10000 \mathrm{~cm}^{2}\right)\right], \quad \mathrm{DAF}=\left[\left(\mathrm{IAF}_{2}-\mathrm{IAF}_{1}\right) /\left(\mathrm{T}_{2}-\mathrm{T}_{1}\right)\right], \quad \mathrm{TCC}=\left[\left(\mathrm{PS}_{2}-\mathrm{PS}_{1}\right) /\left(\mathrm{T}_{2}-\mathrm{T}_{1}\right)\right] \quad \mathrm{y}$ $\mathrm{TAN}=\left\{\left[\left(\mathrm{PS}_{2}-\mathrm{PS}_{1}\right) /\left(\mathrm{AF}_{2}-\mathrm{AF}_{1}\right)\right] /\left[\left(\mathrm{InAF}_{2}-\mathrm{InAF}_{1}\right) /\left(\mathrm{T}_{2}-\mathrm{T}_{1}\right)\right]\right\} ;$ donde: $\mathrm{AF}=$ área foliar, $\mathrm{NP}=$ número de plantas, $\mathrm{DP}=$ densidad de población, $\mathrm{T}=$ tiempo, 1 y $2=$ inicio y final del intervalo, $\mathrm{IAF}=$ índice de área foliar, $\mathrm{PS}=$ peso de MS, InAF= logaritmo natural del área foliar.

\section{Resultados y discusión}

\section{Elementos del clima y etapas fenológicas}

En condiciones de punta de riego, la temperatura mínima fluctuó de 2.3 a $12.6{ }^{\circ} \mathrm{C}$ y la máxima de 23.9 a $31.7{ }^{\circ} \mathrm{C}$, la precipitación acumulada fue $630 \mathrm{~mm}$, donde $42 \%$ (269 mm) ocurrió en fase vegetativa y $58 \%$ (361 mm) en etapa reproductiva (Figura 1).

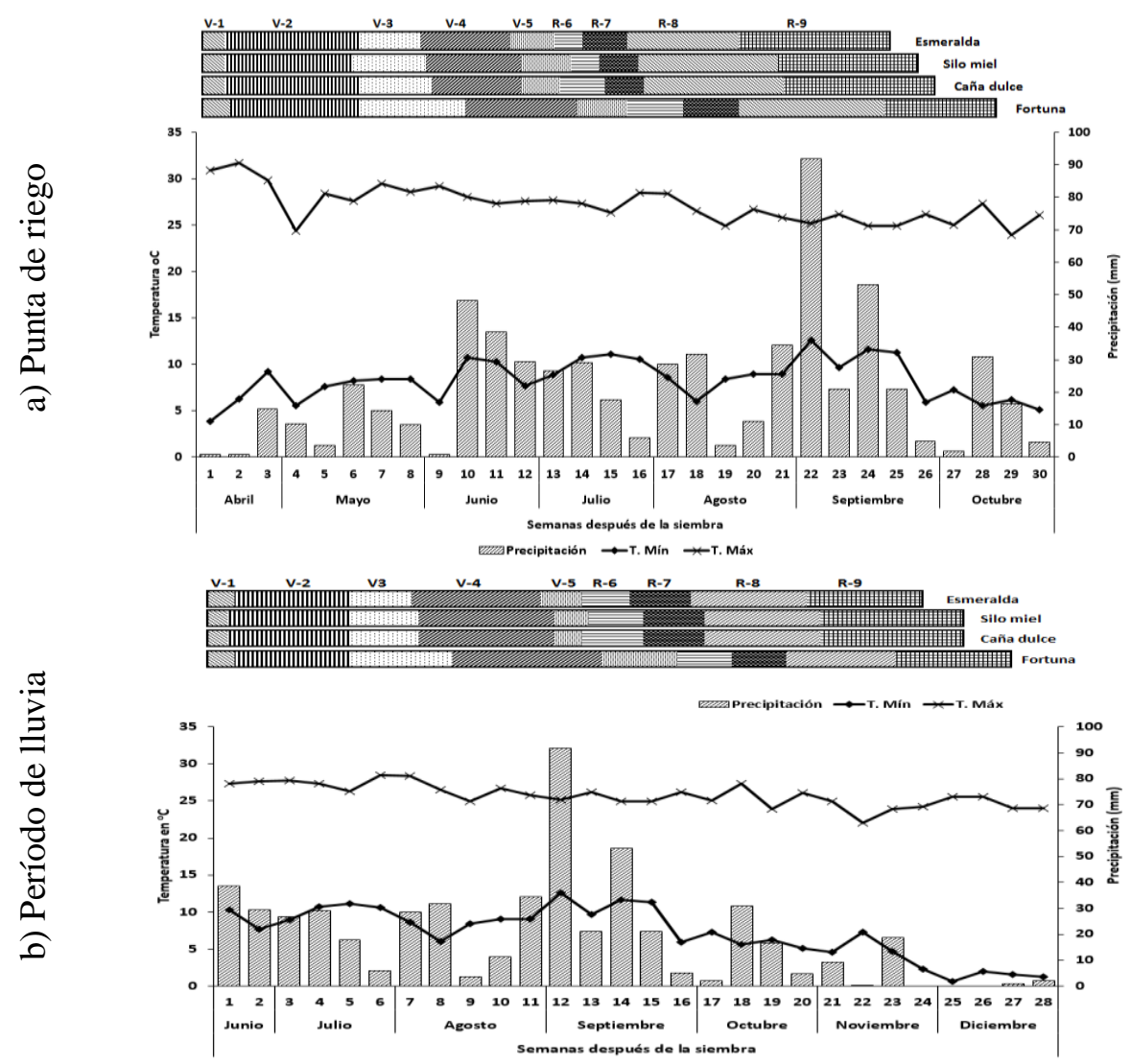

Figura 1. Condiciones climáticas y etapas fenológicas en cuatro variedades de sorgo forrajero, en Montecillo, Estado de México, 2013. 
En período de lluvias, la temperatura mínima fluctuó de $2-12.8^{\circ} \mathrm{C}$ y máxima $24-28.5^{\circ} \mathrm{C}$, por otra parte, la precipitación acumulada fue $534 \mathrm{~mm}$, donde $84 \%$ (444 mm) ocurrió en la fase vegetativa y $16 \%$ (90 mm) en etapa reproductiva. Al respecto Cordovés y Grizel (2009), reportan que el sorgo requiere de 450-650 $\mathrm{mm}$ de precipitación por ciclo de cultivo, por tanto, la precipitación fue adecuada para el desarrollo del cultivo en ambos períodos de siembra; sin embargo, en Texcoco, Estado de México, la temperatura máxima promedio en ambos períodos fue $26^{\circ} \mathrm{C}$, con mañanas frías $\left(8^{\circ} \mathrm{C}\right)$, lo cual limitó el desarrollo del sorgo, dado que la temperatura óptima tiene un rango de $27-29^{\circ} \mathrm{C}$ y las plantas $\mathrm{C} 4$, requieren temperatura mínima de crecimiento de $10{ }^{\circ} \mathrm{C}$.

A pesar de variaciones en humedad y temperatura, la fenología fue semejante para ambos períodos de siembra, la etapa reproductiva inicio con 13 semanas; el cultivar precoz fue Esmeralda, que alcanzó madurez fisiológica en 25 semanas y el cultivar más tardío (Fortuna) en 28 semanas. El intervalo entre etapas fenológicas del sorgo está determinado por genotipo y ambiente, donde la temperatura y precipitación presentan mayor influencia, lo que puede adelantar o alargar el ciclo de vida de las plantas (Solórzano, 2007).

\section{Componentes morfológicos}

Son el resultado de la expresión ambiental, nutricional y genética de las variedades (Solorzano, 2007), donde el carácter de mayor valor económico es el rendimiento de materia seca. En este sentido, el mayor rendimiento fue ligeramente superior en período de riego (Cuadro. 1). La variedad que acumulo la mayor cantidad de biomasa en menor tiempo fue Caña dulce tanto en período de riego (15.7 $\mathrm{t} \mathrm{MS} \mathrm{ha}^{-1}$ ) y temporal (Cuadro 2) $15.5 \mathrm{t} \mathrm{MS} \mathrm{ha}^{-1}$ ), a $145 \mathrm{dds}$ en temporal podríamos cortar a partir de $115 \mathrm{dds}$, debido a que el incremento en materia seca es mínimo, y evitamos deterioro por lignificación. Al respecto Bolaños et al., reporta rendimientos de sorgo forrajero que van de 13.8 a 17.1 t MS año, bajo condiciones de trópico húmedo.

Se observaron diferencias ( $p \leq 0.001$; Cuadro 1 y 2 ) entre variedades, períodos de siembra, AP, NDT, NDH, NDN y RMS, se incrementaron conforme avanzó el ciclo de cultivo en un inicio, el crecimiento fue lento reflejándose en valores pequeños de AP, NDT, NDN y RMS. Al respecto Afzal et al. (2012), evaluaron rendimiento de sorgo forrajero fertilizado con $75 \mathrm{~kg} \mathrm{~N} \mathrm{ha}^{-1}$ e indican que la planta creció $180 \mathrm{~cm}$, como respuesta a la fertilización aumento el número de nudos y distancia entre ellos, reportan 43 tallos por macollo.

Cuadro 1. Componentes morfológicos y rendimiento en sorgo forrajero en período de riego, en Montecillo, Estado de México, 2013.

\begin{tabular}{|c|c|c|c|c|c|c|}
\hline \multirow{2}{*}{ Variedad } & \multirow{2}{*}{ dds } & \multicolumn{4}{|c|}{ Medición por planta } & \multirow{2}{*}{$\begin{array}{c}\text { RMS } \\
\left(\mathrm{t} \mathrm{MS} \mathrm{ha}^{-1}\right)\end{array}$} \\
\hline & & $\mathrm{AP}(\mathrm{cm})$ & NDT & $\mathrm{NDH}$ & NDN & \\
\hline \multirow[t]{5}{*}{ Caña dulce } & 145 & $220.4 \mathrm{a}$ & $46.5 \mathrm{a}$ & $7.7 \mathrm{abc}$ & $8.3 \mathrm{a}$ & $15.7 \mathrm{a}$ \\
\hline & 115 & $220.4 \mathrm{a}$ & $46.5 \mathrm{a}$ & $7.7 \mathrm{abc}$ & $8.3 \mathrm{a}$ & $14.3 \mathrm{~b}$ \\
\hline & 85 & $105.9 \mathrm{~cd}$ & $9 \mathrm{c}$ & $7.7 \mathrm{abc}$ & $3 \mathrm{~d}$ & $7.5 \mathrm{e}$ \\
\hline & 55 & $51 \mathrm{fgh}$ & $5.6 \mathrm{c}$ & 6.5 bcde & $0.5 \mathrm{e}$ & 3.61 \\
\hline & 25 & $31.3 \mathrm{ghi}$ & $3.8 \mathrm{c}$ & 5.7 cde & $0 \mathrm{e}$ & $1.4 \mathrm{k}$ \\
\hline
\end{tabular}




\begin{tabular}{lcccccc}
\hline \multirow{2}{*}{ Variedad } & $\mathrm{dds}$ & \multicolumn{3}{c}{ Medición por planta } & $\begin{array}{c}\text { RMS } \\
\text { (t MS ha }\end{array}$ \\
\cline { 2 - 6 } Silo miel & & $\mathrm{AP}(\mathrm{cm})$ & $\mathrm{NDT}$ & $\mathrm{NDH}$ & $\mathrm{NDN}$ & $14.2 \mathrm{~b}$ \\
& 145 & $146.8 \mathrm{~b}$ & $33.5 \mathrm{a}$ & $9 \mathrm{a}$ & $6.5 \mathrm{bc}$ & $11.8 \mathrm{c}$ \\
& 115 & $146.8 \mathrm{~b}$ & $33.5 \mathrm{a}$ & $9 \mathrm{a}$ & $6.5 \mathrm{bc}$ & $5.6 \mathrm{f}$ \\
& 85 & $97.7 \mathrm{~cd}$ & $4.8 \mathrm{c}$ & $9 \mathrm{a}$ & $3 \mathrm{~d}$ & $3.2 \mathrm{ij}$ \\
Esmeralda & 55 & $59 \mathrm{f}$ & $5 \mathrm{c}$ & $7 \mathrm{abcd}$ & $0.3 \mathrm{e}$ & $1 \mathrm{k}$ \\
& 25 & $26.9 \mathrm{~h}$ & $3 \mathrm{c}$ & $4.8 \mathrm{de}$ & $0 \mathrm{e}$ & $9.5 \mathrm{~d}$ \\
& 145 & $122.9 \mathrm{bc}$ & $31 \mathrm{ab}$ & $8.2 \mathrm{ab}$ & $7.4 \mathrm{a}$ & $9 \mathrm{~d}$ \\
Fortuna & 115 & $122.9 \mathrm{bc}$ & $31 \mathrm{ab}$ & $8.2 \mathrm{ab}$ & $7.4 \mathrm{a}$ & $5 \mathrm{fg}$ \\
& 85 & $85.8 \mathrm{de}$ & $5.5 \mathrm{c}$ & $8.2 \mathrm{ab}$ & $3.3 \mathrm{~d}$ & $0.9 \mathrm{ij}$ \\
& 55 & $52.7 \mathrm{fgh}$ & $3.3 \mathrm{c}$ & $6.6 \mathrm{bcd}$ & $0.3 \mathrm{e}$ & $0.01 \mathrm{k}$ \\
& 25 & 24.11 & $3 \mathrm{c}$ & $4.5 \mathrm{e}$ & $0 \mathrm{e}$ & $4.9 \mathrm{fg}$ \\
& 145 & $95.5 \mathrm{de}$ & $15.8 \mathrm{bc}$ & $7.8 \mathrm{abc}$ & $5.3 \mathrm{c}$ & $4.4 \mathrm{~g}$ \\
Media & 115 & $95.5 \mathrm{de}$ & $15.8 \mathrm{bc}$ & $7.8 \mathrm{abc}$ & $5.3 \mathrm{c}$ & $2.9 \mathrm{~h}$ \\
Variedad & 85 & $69.9 \mathrm{ef}$ & $3.8 \mathrm{c}$ & $7.8 \mathrm{abc}$ & $0.6 \mathrm{e}$ & $0.05 \mathrm{jk}$ \\
Variedad*dds*período & $54.3 \mathrm{fg}$ & $4 \mathrm{c}$ & $7 \mathrm{abcd}$ & $0 \mathrm{e}$ & $0.02 \mathrm{k}$ \\
\hline
\end{tabular}

Medias con distinta literal entre columnas son diferentes estadísticamente Tukey $(\alpha \leq 0.05)$. AP= altura de planta; $\mathrm{NDT}=$ número de tallos por macollo; $\mathrm{NDH}=$ número de hojas en tallo principal; $\mathrm{NDN}=$ número de nudos en tallo principal; RMS $=$ rendimiento de materia seca $\left(\mathrm{t} \mathrm{MS} \mathrm{ha}^{-1}\right)$; significancia ${ }^{*}=p \leq 0.05^{* * *}=p \leq 0.001 ; \mathrm{NS}=$ no significativo.

En el presente trabajo, Caña dulce a 145 dds en ambos períodos de siembra, presenta valores superiores en AP y semejante NDT, en período de riego. El número promedio de hojas y nudos a 145 dds, en ambos métodos de siembra fue semejante; al respecto Kapanigowda et al. (2013), al evaluar 48 líneas de sorgo el número de hojas varió en una por planta.

Las variedades de sorgo presentaron su fase de crecimiento acelerado posterior a 55 dds, que abarca desde la etapa fenológica V-3 a R-8. En la etapa V-3 se dio la elongación de entrenudos, tal como lo reportan Afzal el al. (2012), esto se refleja a 85 dds con mayor AP, NDT, NDH, NDN y RMS (Cuadro 1 y 2). Caña dulce presentó mejores parámetros morfológicos con respecto a las variedades estudiadas, porque está adaptada a condiciones edafoclimáticas del Altiplano Central de México.

Cuadro 2. Componentes morfológicos y rendimiento en sorgo forrajero en período de temporal, en Montecillo, Estado de México, 2013.

\begin{tabular}{|c|c|c|c|c|c|c|}
\hline \multirow{2}{*}{ Variedad } & \multirow{2}{*}{ dds } & \multicolumn{4}{|c|}{ Medición por planta } & \multirow{2}{*}{$\begin{array}{c}\text { RMS } \\
\left(\mathrm{t} \mathrm{MS} \mathrm{ha}^{-1}\right)\end{array}$} \\
\hline & & $\mathrm{AP}(\mathrm{cm})$ & NDT & $\mathrm{NDH}$ & $\mathrm{NDN}$ & \\
\hline \multirow[t]{5}{*}{ Caña dulce } & 145 & $200.1 \mathrm{a}$ & $20 \mathrm{a}$ & $8.5 \mathrm{a}$ & $8.4 \mathrm{a}$ & $15.5 \mathrm{a}$ \\
\hline & 115 & $133.8 \mathrm{bc}$ & $15 \mathrm{abc}$ & $8.4 \mathrm{ab}$ & $7.5 \mathrm{ab}$ & $15.2 \mathrm{ab}$ \\
\hline & 85 & $136.4 \mathrm{~b}$ & $14.5 \mathrm{abc}$ & $8 \mathrm{abc}$ & $6.2 \mathrm{bcd}$ & $7.2 \mathrm{e}$ \\
\hline & 55 & 92.7 bcdefg & $10 \mathrm{abc}$ & $7.5 \mathrm{abcd}$ & $2.2 \mathrm{e}$ & $1.8 \mathrm{hi}$ \\
\hline & 25 & 20.6 hij & $8.3 \mathrm{abc}$ & $6.3 \mathrm{bdd}$ & $0 \mathrm{f}$ & $0.07 \mathrm{j}$ \\
\hline
\end{tabular}




\begin{tabular}{|c|c|c|c|c|c|c|}
\hline \multirow{2}{*}{ Variedad } & \multirow{2}{*}{ dds } & \multicolumn{4}{|c|}{ Medición por planta } & \multirow{2}{*}{$\begin{array}{c}\text { RMS } \\
\left(\mathrm{t} \mathrm{MS} \mathrm{ha}^{-1}\right)\end{array}$} \\
\hline & & $\mathrm{AP}(\mathrm{cm})$ & NDT & $\mathrm{NDH}$ & NDN & \\
\hline \multirow[t]{5}{*}{ Silo miel } & 145 & $133.8 \mathrm{bc}$ & $11.8 \mathrm{abc}$ & $8.3 \mathrm{ab}$ & $7.2 \mathrm{abc}$ & $14.1 \mathrm{~b}$ \\
\hline & 115 & $122.4 \mathrm{bcd}$ & 9 abc & $7.8 \mathrm{abc}$ & $6.3 \mathrm{bcd}$ & $12.3 \mathrm{c}$ \\
\hline & 85 & 108 bcde & $6.8 \mathrm{bc}$ & 7 abcd & $4.8 \mathrm{~d}$ & $5.8 \mathrm{ij}$ \\
\hline & 55 & 70.5 defghij & $7.8 \mathrm{abc}$ & $6.8 \mathrm{abcd}$ & $1.3 \mathrm{ef}$ & $1.2 \mathrm{ij}$ \\
\hline & 25 & $20.6 \mathrm{ij}$ & 7 bc & $5.3 \mathrm{~d}$ & $0 \mathrm{f}$ & $0.04 \mathrm{j}$ \\
\hline \multirow[t]{5}{*}{ Esmeralda } & 145 & $144.9 \mathrm{ab}$ & $16.3 \mathrm{ab}$ & $8 \mathrm{abc}$ & $7.8 \mathrm{ab}$ & $8.8 \mathrm{~d}$ \\
\hline & 115 & 99.8 bcdef & $7.5 \mathrm{bc}$ & $8 \mathrm{abc}$ & $7.2 \mathrm{abc}$ & $8.3 \mathrm{de}$ \\
\hline & 85 & 74 defghi & $4 \mathrm{bc}$ & $7.8 \mathrm{abc}$ & $2.8 \mathrm{e}$ & $4.6 \mathrm{fg}$ \\
\hline & 55 & 50 fghij & $3.3 \mathrm{c}$ & 6 abcd & $1.4 \mathrm{ef}$ & $0.7 \mathrm{ij}$ \\
\hline & 25 & $15.9 \mathrm{j}$ & $3 c$ & $5.8 \mathrm{~cd}$ & $0 \mathrm{f}$ & $0.02 \mathrm{j}$ \\
\hline \multirow[t]{5}{*}{ Fortuna } & 145 & $142 \mathrm{~b}$ & $13.3 \mathrm{abc}$ & $8.4 \mathrm{ab}$ & $7.1 \mathrm{abc}$ & $5.2 \mathrm{fg}$ \\
\hline & 115 & 78.2 cdefgh & $3 \mathrm{c}$ & $7.2 \mathrm{abcd}$ & $5.5 \mathrm{~cd}$ & $4.3 \mathrm{~g}$ \\
\hline & 85 & 51.8 efghij & $3 \mathrm{c}$ & $7.1 \mathrm{abcd}$ & $0.3 \mathrm{~cd}$ & $2.6 \mathrm{~h}$ \\
\hline & 55 & 35.6 ghij & $3 \mathrm{c}$ & $6.3 \mathrm{abcd}$ & $0 \mathrm{f}$ & $0.4 \mathrm{j}$ \\
\hline & 25 & $13.9 \mathrm{j}$ & $3 \mathrm{c}$ & $5.9 \mathrm{~cd}$ & $0 \mathrm{f}$ & $0.02 \mathrm{j}$ \\
\hline Media & & 109.1 & 10.6 & 9.1 & 4.8 & 5.5 \\
\hline Variedad & & $* * *$ & $* * *$ & $*$ & $* * *$ & $* * *$ \\
\hline Variedad $* \mathrm{~d}$ & eríodo & $* * *$ & NS & $* *$ & $* * *$ & $* * *$ \\
\hline
\end{tabular}

Medias con distinta literal entre columnas son diferentes estadísticamente Tukey $(\alpha \leq 0.05)$. AP= altura de planta; $\mathrm{NDT}=$ número de tallos por macollo; $\mathrm{NDH}=$ número de hojas en tallo principal; $\mathrm{NDN}=$ número de nudos en tallo principal; RMS $=$ rendimiento de materia seca $\left(\mathrm{t} \mathrm{MS} \mathrm{ha}^{-1}\right)$. Significancia ${ }^{*}=p \leq 0.05 ;{ }^{* * *}=p \leq 0.001 ; \mathrm{NS}=$ no significativo.

\section{Índice de área foliar (IAF)}

En general las cuatro variedades mostraron incrementó conforme avanzó el ciclo del cultivo, Silo miel presentó mayor IAF a 114 dds con 5 en punta de riego y 6.8 en período de lluvias (Figura 2).

Posteriormente, disminuyó al alcanzar la madurez fisiológica del cultivo, debido a la senescencia foliar y translocación hacia órganos reproductivos (Smart, 1994). Resultados similares encontraron Soto y Hernández (2012), al evaluar Sorghum bicolor (L.) Moench cultivar ISIAP Dorado, donde el valor máximo fue 5.6 a 80 dds, en condiciones de riego. La variedad Silo miel presentó mayor IAF en punta de riego ( 6 a 120 dds) y período de lluvias (7, a 120 dds) y Fortuna, presentó menor valor en punta de riego (4.5, a 125 dds) y período de lluvias (2.9, a 145 dds).

Lo anterior se debió principalmente, a que Silo miel presentó hojas de mayor longitud y anchura, mientras que Fortuna, presentó hojas de menores dimensiones. 

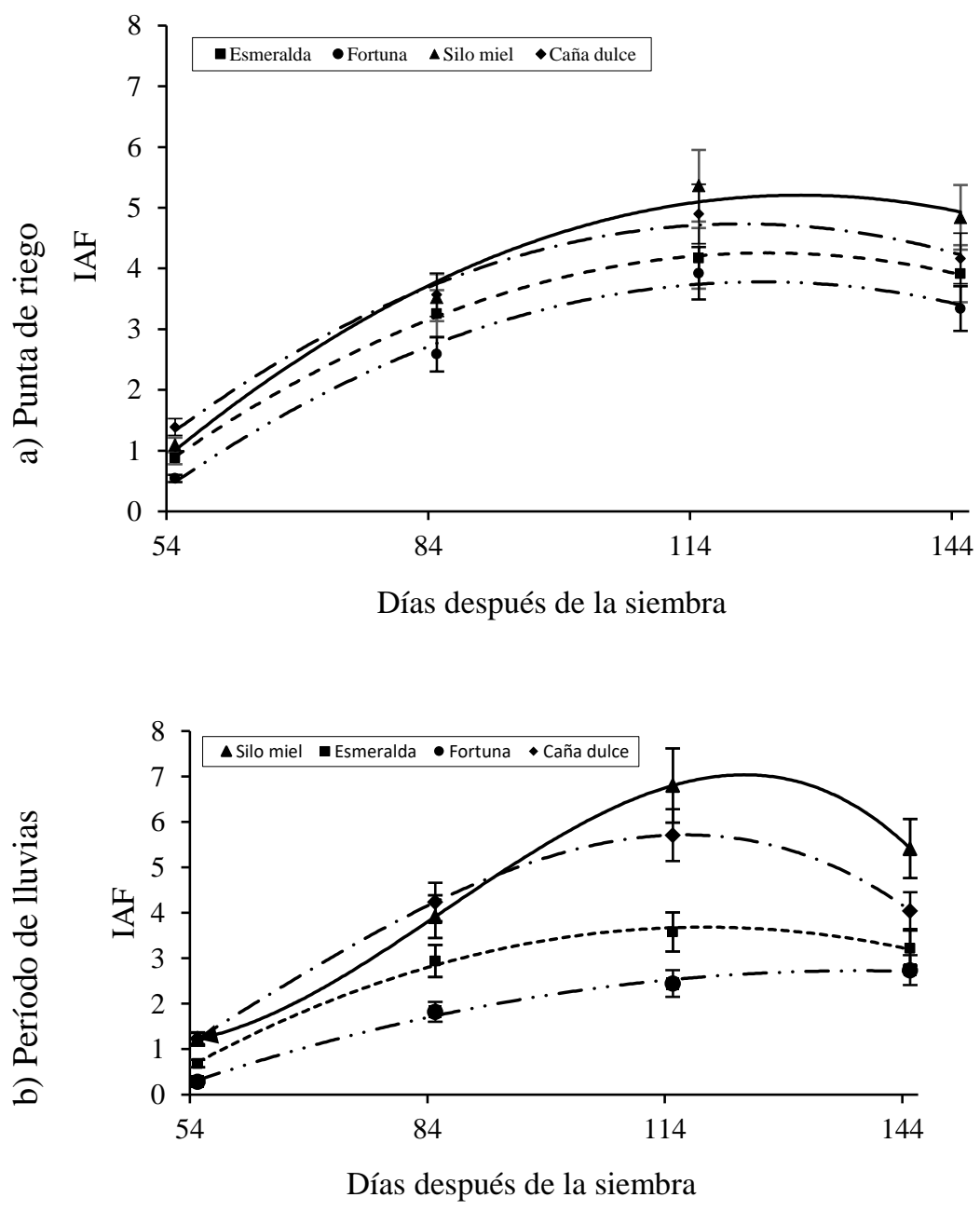

Figura 2. Índice de área foliar (IAF), en cuatro variedades de sorgo en condiciones de punta de riego y temporal, en Montecillo, Estado de México, 2013.

\section{Duración de área foliar}

La máxima DAF se presentó en el cultivar Silo miel con 114 dds en punta de riego y en período de lluvias; lo anterior debido a que en este último período presentó mayor IAF y el dosel vegetal obtiene la mayor radiación interceptada (Figura 3) (Rodríguez y Leihner, 2005), mientras que Fortuna presentó menor DAF a 110 días en punta de riego a 80 días en condiciones de lluvias.

El IAF es tan importante como la DAF, a medida que esta es más efectiva en el tiempo, implica mayor eficiencia en aprovechamiento de radiación solar; lo cual, se manifiesta en un mayor crecimiento y acumulación de materia seca durante el ciclo del cultivo (Hernández y Soto, 2013). 


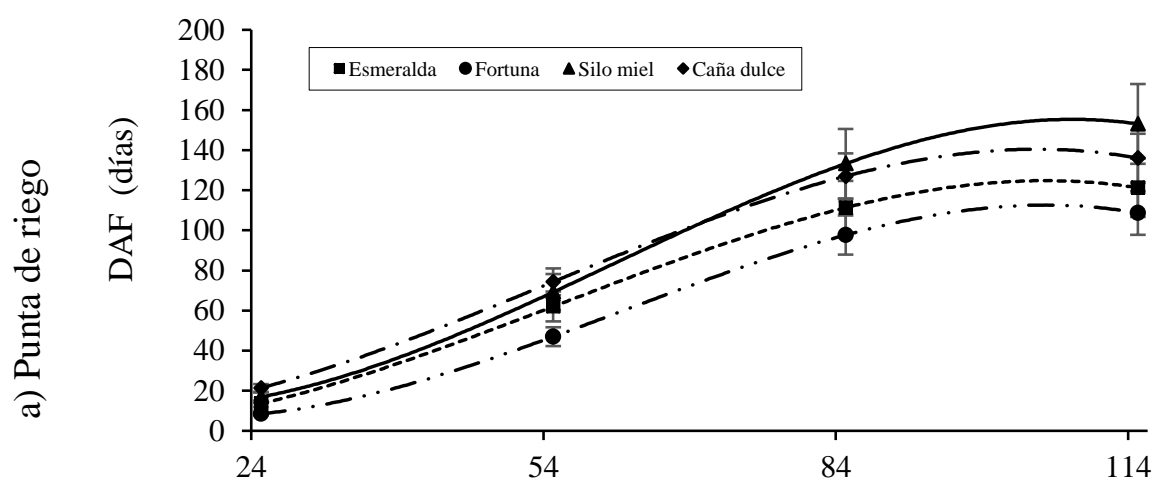

Días después de la siembra

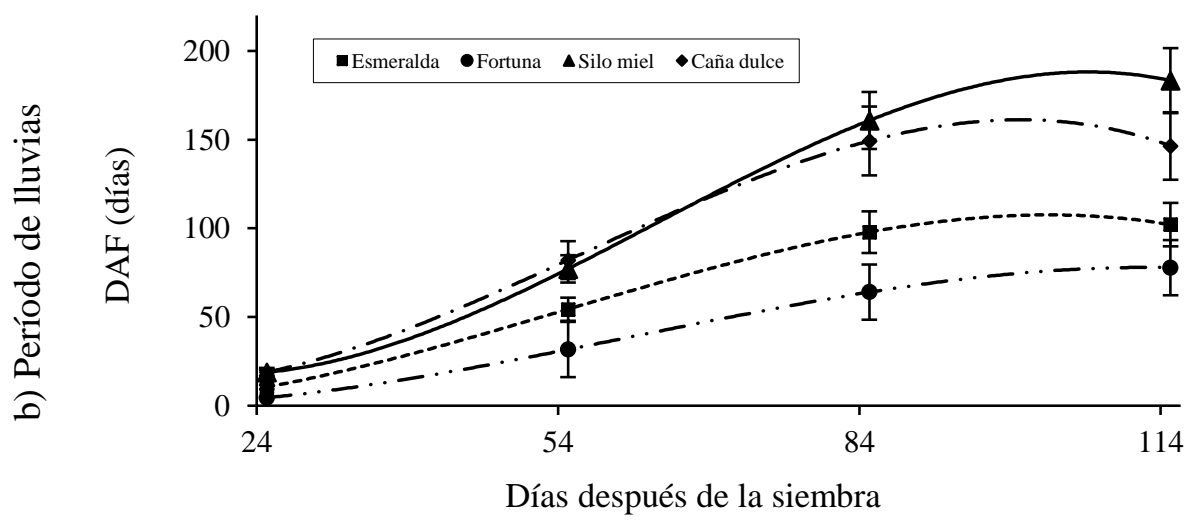

Figura 3. Duración de área foliar (DAF), en cuatro variedades de sorgo en condiciones de punta de riego y temporal, en Montecillo, Estado de México, 2013.

\section{Tasa de crecimiento del cultivo (TCC)}

En general, para las cuatro variedades se observó un incremento de la emergencia a 25 dds (6.2 g $\mathrm{m}^{-2} \mathrm{~d}^{-1}$, Figura 4).

La TCC alcanzó un máximo en intervalo 56-85 dds $\left(15.4 \mathrm{~g} \mathrm{~m}^{-2} \mathrm{~d}^{-1}\right)$ punta de riego; en período de lluvias, en el intervalo de emergencia a $25 \mathrm{dds}$ presentó TCC de $4.3 \mathrm{~g} \mathrm{~m}^{-2} \mathrm{~d}^{-1}$ y máxima en intervalo de 56-85 dds (16.95 $\left.\mathrm{g} \mathrm{m}^{-2} \mathrm{~d}^{-1}\right)$, posteriormente disminuyó en ambos períodos de siembra, debido a la disminución en crecimiento de las plantas, senescencia de hoja y reducción de fotosíntesis (Gutiérrez et al., 2005). El cultivar Caña dulce presentó mayor TCC, con $24 \mathrm{~g} \mathrm{~m}^{-2} \mathrm{~d}^{-1}$ en intervalo 56-85 dds en punta de riego y $26 \mathrm{~g} \mathrm{~m}^{-2} \mathrm{~d}^{-1}$ en período de lluvias. Fortuna presentó menor TCC con $7.5 \mathrm{~g} \mathrm{~m}^{-2} \mathrm{~d}^{-1}$ (punta de riego) y $8 \mathrm{~g} \mathrm{~m}^{-2} \mathrm{~d}^{-1}$ (lluvias) en intervalo 26-55 dds. Carrillo y Ruiz (2004), obtuvieron resultados similares al evaluar seis variedades de sorgo forrajero en condiciones de riego, el cultivar Dine a Mite alcanzó mayor TCC con $27.9 \mathrm{~g} \mathrm{~m}^{-2} \mathrm{~d}^{-1}$ y menor el cultivar Grazer con $24.42 \mathrm{~g} \mathrm{~m}^{-2} \mathrm{~d}^{-1}$ a $58 \mathrm{dds}$. 


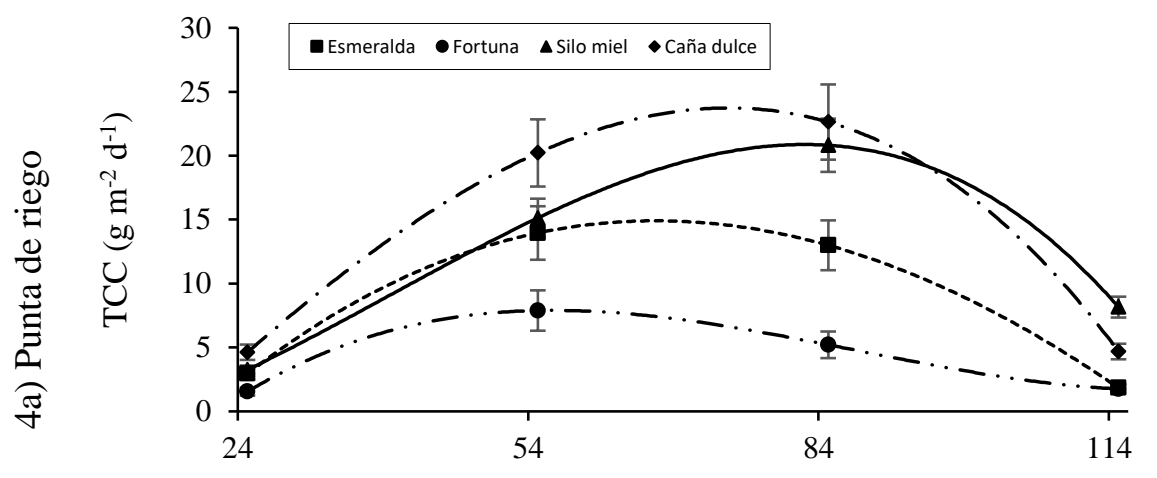

Días después de la siembra

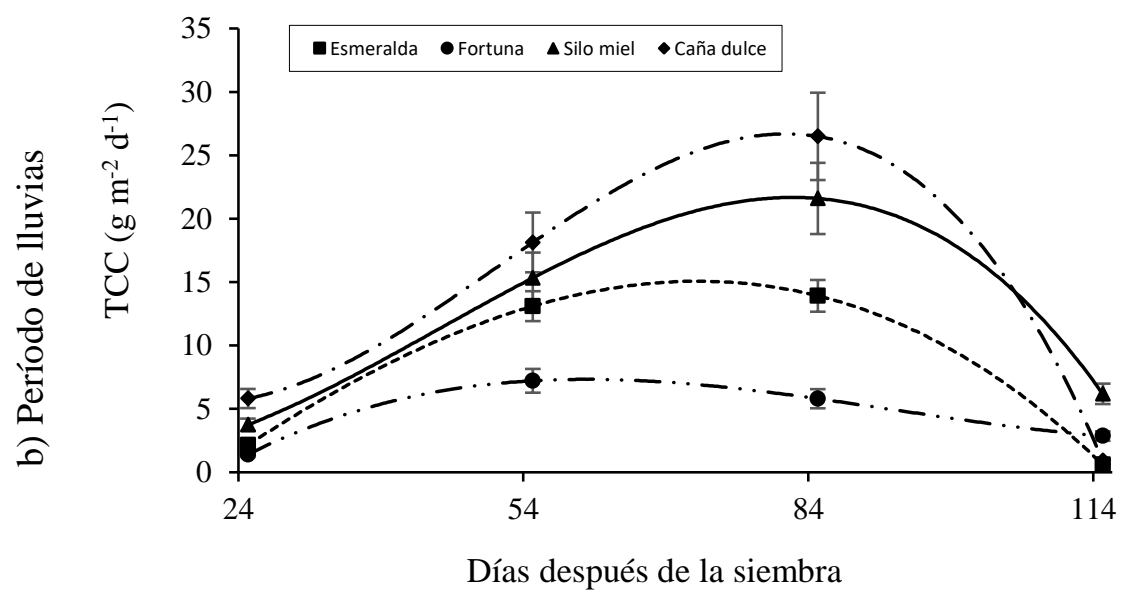

Figura 4. Tasa de crecimiento del cultivo (TCC), en cuatro variedades de sorgo en condiciones de punta de riego y temporal, en Montecillo, Estado de México, 2013.

\section{Tasa de asimilación neta (TAN)}

La TAN en cuatro variedades de sorgo, bajo dos condiciones de producción, se observó que los valores más altos ocurrieron al inicio de la emergencia en el intervalo de 0 a 54 dds y disminuyeron conforme avanzó el ciclo de desarrollo del cultivo, hasta alcanzar madurez fisiológica, cuando se presentaron valores más bajos, esto se debió a competencia por luz, nutrientes, aumento del dosel vegetal, mayor sombreo y senescencia de hojas inferiores (Naresh y Singh, 2001; Figura 5).

En condición de punta de riego, el cultivar Caña dulce presentó mayor TAN del período de emergencia a $25 \mathrm{dds}\left(19.1 \mathrm{~g} \mathrm{~m}^{-2} \mathrm{~d}^{-1}\right), 26-55 \mathrm{dds}\left(7.3 \mathrm{~g} \mathrm{~m}^{-2} \mathrm{~d}^{-1}\right)$ y $56-85 \mathrm{dds}\left(5.4 \mathrm{~g} \mathrm{~m}^{-2} \mathrm{~d}^{-1}\right)$. En período de lluvias ocurrió algo similar, el cultivar Caña dulce presentó los valores más elevados del período de emergencia a $25 \mathrm{dds}\left(19 \mathrm{~g} \mathrm{~m}^{-2} \mathrm{~d}^{-1}\right), 26-55 \mathrm{dds}\left(7 \mathrm{~g} \mathrm{~m}^{-2}\right.$ día $\left.^{-1}\right)$ y $56-85 \mathrm{dds}(5.4 \mathrm{~g}$ $\left.\mathrm{m}^{-2} \mathrm{~d}^{-1}\right)$. 

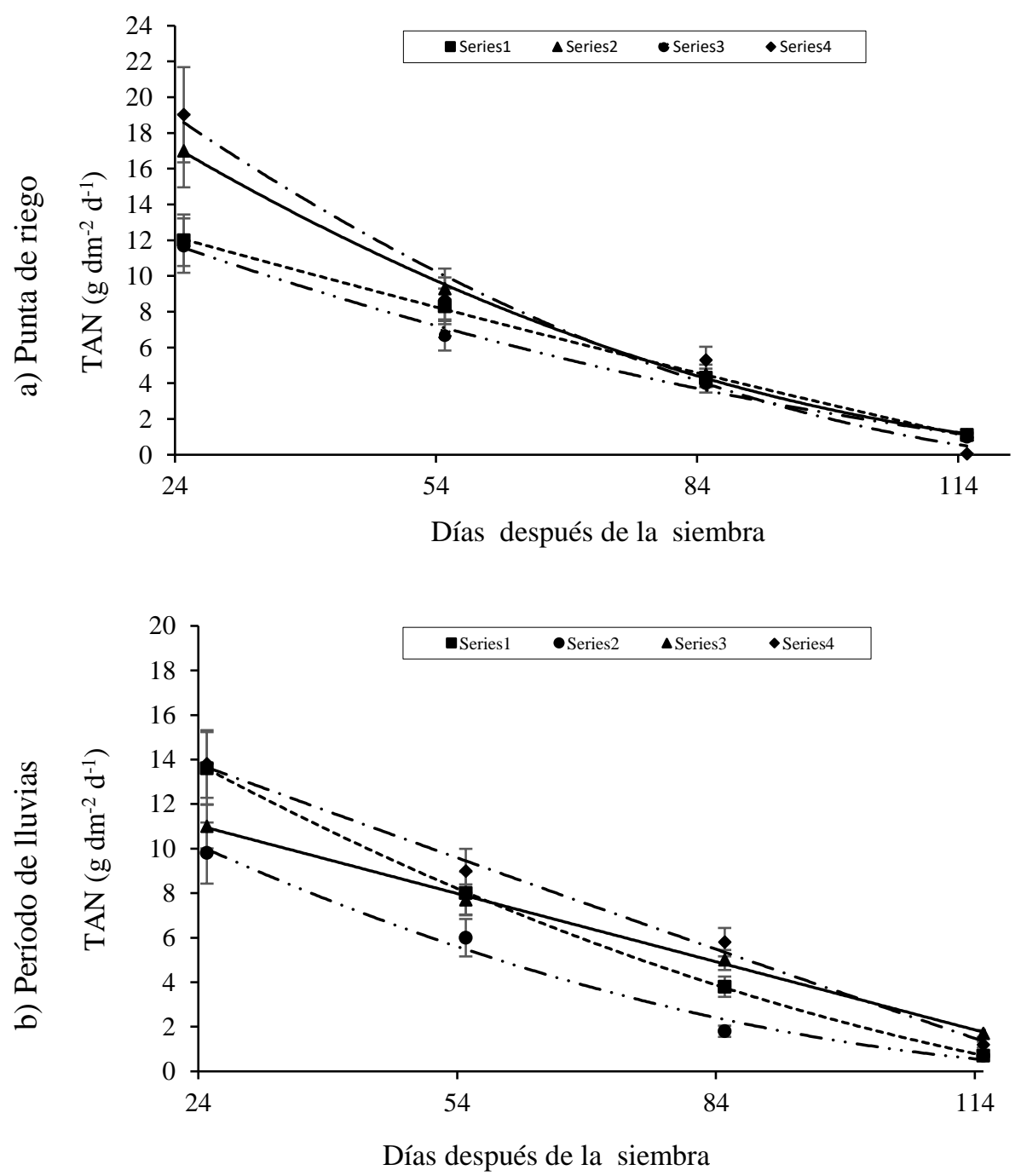

Figura 5. Tasa de asimilación neta (TAN), en cuatro variedades de sorgo en condiciones de punta de riego y temporal, en Montecillo, Estado de México, 2013.

El cultivar Fortuna presentó menor TAN, en todos los intervalos y ambos períodos de siembra (punta de riego y período de lluvias), valores similares reportaron Saberi y Siti (2013), niveles de $\left(6 \mathrm{~g} \mathrm{~m}^{-2} \mathrm{~d}^{-1}\right)$ a los $50 \mathrm{dds}$. Mientras que Carrillo y Ruiz (2004), reportaron TAN a 58 dds en seis variedades evaluadas, con valores que van de $\left(5.17 \mathrm{~g} \mathrm{~m}^{-2} \mathrm{~d}^{-1}\right)$ en el cultivar Beefbuilder-T hasta $\left(8.12 \mathrm{~g} \mathrm{~m}^{-2} \mathrm{~d}^{-1}\right)$ en el cultivar Grazer en condiciones de riego.

Al tratarse de un cultivo con capacidad de amacollamiento, Sorghum bicolor distribuye la producción de forraje total anual en más de un corte, por lo que la siembra a punta de riego tiene la ventaja de cosechar forraje, antes de madurez fisiológica del grano y aprovechar las temperaturas adecuadas para el crecimiento de agosto a octubre, para producir un segundo corte; lo anterior, no ocurre en cultivos tradicionales del altiplano como el maíz. Sorghum bicolor es una buena opción para obtener forraje fresco en dos cosechas, cuando se utilizan siembras a punta de riego. 


\section{Conclusiones}

El cultivar Esmeralda presentó el ciclo de cultivo más corto; sin embargo, el cultivar que presentó mayor rendimiento de materia seca, altura de planta, número de tallos por macollo, número de hojas, número de nudos fue Caña dulce, a 145 días después de la siembra. Los mejores índices de tasa de crecimiento de cultivo y tasa de asimilación neta se observaron en Caña dulce; sin embargo, Silo miel presentó mayor índice de área foliar y duración de área foliar, por lo tanto, el mejor cultivar para las condiciones de Montecillos fue Caña dulce. Al sembrar con 'punta de riego' el sorgo forrajero, se pueden aprovechar de dos a tres cosechas al año y tener una mejor distribución de la materia seca, en el Altiplano Central de México.

\section{Agradecimientos}

Al Consejo Nacional de Ciencia y Tecnología (CONACYT), por la beca de posgrado otorgada a Alejandra Pérez Hernández (506812), lo que permitió la realización de esta investigación.

\section{Literatura citada}

Afzal, M.; Ahmad, A. and Ahmad, A. U. H. 2012. Effect of nitrogen on growth and yield of sorghum forage (Sorghum bicolor (L.) Moench CV). Under three cuttings system. Agronomical research in Moldovia. 45(4):57-64.

Ajeigbe, H. A.; Akinseye, F. M.; Ayuba, K. and Jonah, J. 2018. Productivity and water use efficiency of sorghum [(Sorghum bicolor (L.) Moench.], grown under different nitrogen applications in Sudan savanna zone, Nigeria. Intl. J. Agron. https://doi.org/10.1155/2018/7676058.

Azam, A. S. 2013. Plant and crop science. In: Gregory P. J. and S. Nortcliff (Eds.). Soil conditions and plant growth. wiley blackwell. Oxforf, UK. 22-46 pp. https://marwanbaloch.files.wordpress.com/2015/01/soil-conditions-and-plant-growth.pdf.

Bednarz, C. W.; Bridges, D. C. and Brown, S. M. 2000. Analysis of cotton yield stability across population densities. Agron. J. 92:128-135.

Bolaños, A. E.; Claude. E. J. y Guillaume, A. 2012. Rendimiento y calidad de híbridos de sorgo con y sin nervadura café*. Rev. Mex. Cienc. Agríc. 3(2):37-43.

Bolaños, A. E. D. y Emile, J. C. 2013. Efecto de la distancia entre surcos y densidad de siembra en el rendimiento y calidad del forraje de sorgo. Rev. Mex. Cienc. Pec. 4(2):161-176.

Borghi, E.; Crusciol, C. A. C.; Nascentec, A. S.; Sousa, V. V.; Martins, P. O.; Mateus, G. P. and Costa, C. 2013. Sorghum grain yield, forage biomass production and revenue as affected by intercropping time. Eur. J. Agron. 51:130-139.

Carrillo, J. C. y Ruiz, J. 2004. Producción de forraje en sorgo y mijo: variables de crecimiento. Agron. Mesoam. 15(1):69-76.

Castro, J.; Ortíz, J.; Mendoza, M. C. y Zavala, F. 2000. Producción de biomasa en líneas de sorgo como respuesta al estrés hídrico. Rev. Fitotec. Méx. 23:321-334.

Cordovés, B. G. y Grizel, M. D. 2009. Sorgo dulce: sus potencialidades productivas. ICIDCA. Sobre los derivados de la caña de azúcar. 48:15-21.

Di, R. J. A.; Casanoves, F.; Balzarini, M. G.; González, M.G.; Tablada, M. y Robledo, C. W. 2008. InfoStat. Universidad Nacional de Córdoba, Argentina. 336 p.

Egli, D. B. 2017. Seed biology and yield of grain crops. $2^{\text {nd }}$ Edition. CABI. Wallingfor, UK. 232 p. 
Escalante, E. J. A. y Kohashi, S. J. 2015. El rendimiento y crecimiento del frijol. Manual para la toma de datos. Colegio de Postgraduados. Montecillo, Texcoco, Estado de México. 84 p.

García, E. 2005. Modificaciones al sistema de clasificación climática de Köppen. Cuarta Edición. Universidad nacional Autónoma de México (UNAM). DF. 217 p.

Gaytán, M. A.; Palomo, G. A. y Godoy, A. S. 2001. Eficiencia en la producción y distribución de biomasa en variedades precoces de algodón. Rev. Fitotec. Mex. 24:197-202.

Granados, R. B. y Sarabia, R. A. A. 2013. Cambio climático y efectos en la fenología del maíz en el DDR-Toluca. Rev. Mex. Cienc. Agríc. 4(3):435-446.

Gutiérrez, R. M.; Paul, R. M.; Escalante, E. J. A. y Larqué, S. A. 2005. Algunas consideraciones en la relación entre fotosíntesis y el rendimiento de grano en trigo. Ciencia Ergo Sum. 12(2):149-154.

Hernández, C. N. y Soto, C. F. 2013. Determinación de índices de eficiencia en los cultivos de maíz y sorgo establecidos en diferentes fechas de siembra y su influencia en el rendimiento. Cultivos Tropicales. 34:24-29.

Hunt, R. 1990. Basic Growth Analysis. Plant Growth Analysis. Unwing Hyman. London. 112 p.

Kapanigowda, M. H.; Perumal, R.; Aiken, M. R.; Herald, T. J.; Bean, S. R. and Little, C. R. 2013. Analyses of sorghum [Sorghum bicolor (L.) Moench] lines and hybrids in response to early season planting and cool conditions. Can. J. Plant Sci. 93:773-784.

Legarto, J. 2000. L'utilisation en ensilage plante entière des sorghos grains et sucriers: intérêts et limites pour les régions sèches. Fourrages. 163:323-338.

Naresh, K. S. and Singh, C. P. 2001. Growth analysis of maize during long and short duration crop seasons: Influence of nitrogen source and dose. Indian J. Agric. Res. 35:13-18.

Pérez, H. A.; Quero, C. A. R.; Escalante, E. J. A. S.; Rodríguez, G. M. T.; Garduño, V. S. y Miranda, J. L. 2018. Fenología, biomasa y análisis de crecimiento en variedades de sorgo forrajero en valles altos. Agron. Costarric. 42(2):107-117.

Rodríguez, W. y Leihner, D. 2005. Análisis de crecimiento vegetal. Fisiología de los cultivos tropicales. Ed. UCR. Costa Rica. 39 p.

Rouf, A. D.; Eajaz, A. D.; Ajit, K. and Urmila, G. P. 2018. Sweet sorghum-a promising alternative feedstock for biofuel production. Renewable and Sustainable Energy Reviews. 82(3):4070-4090.

Saberi, A. R and Siti, A. H. 2013. Growth analysis of forage Sorghum (Sorghum bicolor L.) varieties under varying salinity and irrigation frequency. Inter. J. Biotechnol. 2(7):130-140.

SIAP. 2018. Servicio de Información Agroalimentaria y Pesquera, México. Avance de siembras y cosechas, resumen por cultivo (Sorgo). Ciudad de México, México. http://infosiap.siap.gob.mx:8080/ agrícola_siap_gobmx/ resumendelegacion.do.

Smart, C. 1994. Gene expression during leaf senescence. New Phytologist. 126:419-448.

Solórzano, E. V. 2007. Guías fenológicas para cultivos básicos. Ed. Trillas. México, DF. 152 p.

Soto, C. F. y Hernández, C. N. 2012. Influencia de tres fechas de siembra en el crecimiento y rendimiento de especies de cereales cultivadas en condiciones tropicales. Parte II. Cultivo del sorgo (Sorghum bicolor L. Moench var. Isiap Dorado). Cultivos Tropicales. 33:50-54. 